\title{
Effective Elimination of Lymphoma Cells in Stromal Microenvironment by Double Impact on ROS and ATP Metabolism Using Auranofin
}

\author{
Mengqi Yang \\ Sun Yat-sen University Cancer Center \\ Panpan Liu \\ Sun Yat-sen University Cancer Center \\ Huanting Chen
}

People's Hospital of Bao'an District, Shenzhen

Jing Yang

Sun Yat-sen University Cancer Center

\section{Shijun Wen}

Sun Yat-sen University Cancer Center

Yumin Hu

Sun Yat-sen University Cancer Center

Wenhua Lu

Sun Yat-sen University Cancer Center

\section{Ping Chen}

Sun Yat-sen University Cancer Center

Guoxin Hou

Sun Yat-sen University Cancer Center

Weiye Jiang

Sun Yat-sen University Cancer Center

Wenqi Jiang

Sun Yat-sen University Cancer Center

Jinyun Liu ( $\square$ liujinyun@sysucc.org.cn )

Sun Yat-sen University Cancer Center

Peng Huang

Sun Yat-sen University Cancer Center

\section{Research}

Keywords: Auranofin, Thioredoxin reductases, Lymphoma, Stromal microenvironment, Cancer metabolism 
Posted Date: August 11th, 2020

DOI: https://doi.org/10.21203/rs.3.rs-55280/v1

License: (c) (i) This work is licensed under a Creative Commons Attribution 4.0 International License. Read Full License 


\section{Abstract \\ Background}

Protection of cancer cells by the stromal microenvironment is an important drug-resistant mechanism in vivo, and poses a major challenge in cancer treatment. Based on our recent findings that auranofin (AF), an anti-rheumatic drug known to inhibit thioredoxin reductases (TrxR), was effective against several cancer models in vivo, this study tested the ability of AF to eliminate cancer cells in the tumor stromal microenvironment and investigated the underlying mechanisms.

\section{Methods}

Cytotoxic effect of AF on 33 cancer cell lines in vitro was determined by MTS assay to identify AFsensitive cancer cells. Further cytotoxic effect of AF on the identified cancer cells was determined by flow cytometry analysis in stromal co-culture system. Mitochondrial bioenergetics assays using the Seahorse XF24 analyzer, mass spectrometry analyses, and enzyme activity assays were used in the mechanistic study. For in vivo study, lymphoma xenograft models were utilized to evaluate the in vivo therapeutic activity of AF in mice.

\section{Results}

We unexpectedly found that AF exhibited higher therapeutic activity against lymphoma cells in stromal microenvironment compared to lymphoma cell treated with AF without stromal cells. Mechanistic study showed that AF impacted energy metabolism by inhibiting the glycolytic enzyme GAPDH and suppressing mitochondrial electron transport complex II, leading to ATP depletion. Surprisingly, inhibition of TrxR seemed not critical for its anticancer activity, as genetic silencing of TrxR1 and TrxR2 did not significantly affect cell viability. Co-culture of lymphoma cells with stromal cells enhanced the expression of TrxR1, which became an ROS-generating molecule when interacting with AF. The AF-induced ATP depletion plus ROS generation together effectively killed the cancer cells in stromal microenvironment, and significantly suppressed tumor growth in mice bearing mantle cell lymphoma xenografts.

\section{Conclusions}

Our study reveals a novel mechanism by which AF eliminates cancer cells in tumor microenvironment by impacting cancer metabolism and promoting ROS generation, and suggests a possibility to repurpose this anti-rheumatic drug for treatment of lymphoma and overcome drug resistance in stromal microenvironment.

\section{Introduction}


Despite significant progress in our understanding of cancer biology and in the development of novel therapeutic agents, many cancers remain incurable. One of the major challenges is the development of drug resistance, which often leads to persistence of residual disease and cancer recurrence. Tumor microenvironment plays a major role in promoting cancer cell survival and drug resistance by providing protective tissue niches for cancer cells (1). For instance, some drug-resistant malignant B cells often remains in tissue niches such as lymph nodes and bone marrow. This residual malignant $B$ cells eventually regrow leading to relapse of disease, which is often no longer responsive to original treatment (2). Thus, identification of drugs that are able to effectively kill cancer cells in tumor microenvironment is extremely important to enhance in vivo therapeutic activity and improve clinical outcome.

Auranofin (AF) is a gold-containing drug used in clinical treatment of rheumatoid arthritis (3). Recent studies suggest that this compound also has anticancer activity in both solid tumors and hematologic malignancies $(4,5)$. However, the exact mechanisms responsible for its anti-cancer effect remain poorly understood. Inhibition of TrxRs has been considered as a key pharmacological action of AF, which could result in an increase of reactive oxygen species (ROS) leading to damage to cancer cells (6). However, some studies suggest that oxidative stress induced by AF might not be a key mechanism responsible for its cytotoxic action against cancer cells $(7,8)$. In this study, we found that AF exhibited a potent anticancer activity against various types of cancer cells, and was particularly effective in killing lymphoma cells in the presence of stroma cells mimicking tissue microenvironment, which otherwise would protect cancer cells against traditional anticancer drugs. Further mechanistic study revealed that disruption of cancer metabolism by double hits on ATP generation and direct stimulation of TrxR to produce ROS in cancer cells in the tissue niches by AF, rather than direct inhibition of TrxRs, appear to be the underlying mechanisms of action.

\section{Methods}

Reagents: N-acetyl-L-cysteine (NAC), ascorbate, digitonin, TMPD, succinate, NADPH, TrxR1, oxaliplatin, fludarabine, catalase, purified GAPDH from human erythrocytes, and3-(4,5-dimethylthiazol-2-yl)-5(3carboxymethoxyphenyl)-2-(4-sulfopheny)-2 h-tetrazolium(MTS) were supplied by Sigma-Aldrich (St. Louis, Missouri, USA). Trx-1 was purchased from R\&D Systems (Minneapolis, MN, USA). Sodium pyruvate was obtained from Gibco (Grand Island, NY, USA). Apoptosis detection kit was obtained from KeyGen Biotech (Nanjing, China). Hydroethidine, CM- $\mathrm{H}_{2}$ DCFDA, Rhodamine-123, Nonylacridine orange (NAO), Lipofectamine $^{\text {TM }}$ RNAiMAX were obtained from Invitrogen Corporation (Carlsbad, CA, USA). Human siRNA against TrxR1 and TrxR2 was purchased from Ribobio Company Limited (Guangzhou, China). Auranofin was purchased from Cayman Chemical Company (Ann Arbor, Michigan, USA). It was dissolved in dimethyl sulfoxide (DMSO) as a stock solution and stored in aliquots at $-20^{\circ} \mathrm{C}$.Antibodies were purchased from Abcam (Cambridge, MA, USA).

Flow cytometric analysis: The cells were stained with Annexin-V-FITC/PI for analysis of cell viability using flow cytometer (Beckman Counter, Fullerton, CA, USA).Cells were stained with hydroethidine $(100 \mathrm{ng} / \mathrm{ml}, 1$ hour) for detection of superoxide, $\mathrm{CM}-\mathrm{H}_{2} \mathrm{DCFDA}(5 \mu \mathrm{M}, 40$ minutes) for detection of general ROS, 
Rhodamine-123 (1 $\mu \mathrm{M}, 40$ minutes) for detection of mitochondrial transmembrane potential, or NAO ( $1 \mu \mathrm{M}, 30$ minutes) for detection of mitochondrial cardiolipin, using the manufacturer's recommended conditions and analyzed by flow cytometry.

Cancer-stromal cell co-culture: Stromal cells were seeded in 24-well or 6-well plates, allowed to adhere and grow overnight before addition of tumor cells at a ratio of 3:1 (lymphoma:NKtert cells).

Mitochondrial bioenergetics assay: Suspension cells were immobilized on culture plates coated with CellTak (BD Bioscience, $C N$ ) at a density of $2-3 \times 10^{5}$ cells/well. Oxygen consumption rate (OCR) and extracellular acidification rate (ECAR) were measured in unbuffered DMEM (Sigma-Aldrich) supplemented with $10 \mathrm{mM}$ D-glucose, $2 \mathrm{mM}$ L-glutamine, and/or $1 \mathrm{mM}$ pyruvate as indicated, using a Seahorse XF24 analyzer.

Enzyme activity assays: The cellular TrxR activity was measured using a TrxR Activity Colorimetric Assay kit according to the protocol provided by the manufacturer (Biovision, Milpitas, CA, USA). The activities of mitochondrial respiratory complexes were measured using Complex I/II/III/IV/V Enzyme Activity Microplate Assay Kit (Abcam, Cambridge, MA, USA). GAPDH activity was analyzed using a GAPDH Assay Kit (Scien Cell Research Laboratories, Carlsbad, CA, USA). Cellular hexokinase activity was measured using a Hexokinase Assay Kit (Scien Cell Research Laboratories, Carlsbad, CA, USA) according to the manufacturer's instructions. Pyruvate kinase activity was detected with a Pyruvate Kinase Activity Fluorometric Assay Kit (Biovision, Milpitas, CA, USA). LDH activity was measured by a Lactate Dehydrogenase Activity Colorimetric Assay Kit (Biovision, Milpitas, CA, USA).

In vivo animal studies: JeKo-1 cells were inoculated subcutaneously $\left(2 \times 10^{6}\right.$ cells in matrigel per injection) on the right flanks of 8-week old nude mice ( $\mathrm{n}=10$ mice per group). Four weeks after tumor inoculation, one group of mice received $10 \mathrm{mg} / \mathrm{kg}$ AF by intraperitoneal (i.p.) injection daily, 5 days per week (Monday-Friday). Normal saline was used as a control. After 1 month, the treatment was stopped and the mice were sacrificed. Tumor weights were recorded. To test the effect of AF on cancer cell metabolism in vivo, $2 \times 10^{6}$ JeKo-1 cells were inoculated subcutaneously on the right flanks of nude mice $(\mathrm{n}=3 \mathrm{mice} / \mathrm{group} \times 2$ ). One group received $10 \mathrm{mg} / \mathrm{kg} \mathrm{AF}$ by i.p. injections for 3 days after 4 weeks. Another group of mice were treated with normal saline as control. The mice were sacrificed and lymphoma cells were isolated from the tumor tissues for analysis of OCR and ECAR. All animal experiments were conducted in accordance with the institutional guidelines and approved by the Animal Care and Use Committee of Sun Yat-Sen University Cancer Center. The investigators were not blinded to the group allocation during the experiments.

Statistical analysis: Additional experimental procedures are provided under Supplemental Information. Data were representative of at least three independent experiments unless otherwise indicated. Statistical analyses including two-sided Student's $t$-test and analysis of variance (ANOVA) followed by NewmanKeuls multiple-comparison test were performed using GraphPad Prism software (GraphPad, San Diego, CA, USA). Data are expressed as mean \pm standard deviation (SD). A $p$ value of less than 0.05 is 
considered statistically significant. Group sizes for mice were chosen based on literature and prior experience with these mouse models (9).

\section{Results}

\section{AF selectively kills cancer cells with minimum toxicity to normal cells}

We first tested the sensitivity of various cancer cell lines of different tissue origins to AF. This drug exhibited potent growth inhibition on all 33 cancer cell lines tested, with $I_{50}$ values of less than $2 \mu \mathrm{M}$ in most cases (Fig. 1a). AF was particularly effective against lymphoma cells, including mantle cell lymphoma (MCL) JeKo-1 cell line, primary lymphoma cells isolated from patients with MCL and diffused large B cell lymphoma (DLBCL) (Fig. 1b). Notably, AF at low concentrations (0.2-0.5 $\mu \mathrm{M})$ was highly effective against the malignant cells. In contrast, $A F$ induced minimal cytotoxicity in non-cancerous cells, including primary lymphocytes isolated from the biopsy samples of patients with benign lymphadenitis (Fig. 1b), normal peripheral blood mononuclear cells (PBMC) and normal stromal cells (Fig. 1c and Supplemental Fig. 1a-b).

\section{AF is highly effective against lymphoma cells in the presence of stromal cells}

Since previous studies showed that stromal cells in tumor microenvironment could protect leukemic cells and promote drug resistance (10-13), we tested the effect of AF against lymphoma cells in the presence of stromal cells using a co-culture system. Surprisingly, instead of seeing a protective effect, we found that AF exhibited greater cytotoxic activity against lymphoma cells (both JeKo-1 and Raji) when they were co-cultured with NKtert stromal cells (Fig. 2a-b). These data together show a striking ability of AF to kill malignant cells in stromal microenvironment.

\section{Inhibition of TrxRs by AF is not a key mechanism responsible for cytotoxicity}

It has been suggested that inhibition of TrxRs by AF might lead to ROS-mediated apoptosis (14). We tested the impact of AF on TrxR activity and cell viability in two lymphoma cell lines, and found that AF could indeed inhibit TrxR activity and induced an increase of ROS (Fig. 3a). Pretreatment of cells with an antioxidant $\mathrm{N}$-acetyl-L-cysteine (NAC) seemed able to reduce AF-induced apoptosis (Fig. $3 \mathrm{~b}$ and Supplementary Fig. 2a), suggesting a potential role of ROS in drug-induced cell death. However, since NAC is a thiol-containing compound capable of directly conjugating with certain compounds including AF $(15,16)$, we tested if the protective effect of NAC was due to its conjugation with AF to prevent cellular uptake of AF. Quantitative assay of cellular gold (Au) content showed an $80 \%$ decrease of intracellular AF in the presence of NAC (Fig. 3b and Supplementary Fig. 2b). When the pre-incubated NAC was removed by washing before addition of AF to avoid direct NAC-AF binding in the medium, such washing restored cellular uptake of AF (Fig. 3b and Supplementary Fig. 2b) and almost completely abolished the NAC protective effect against AF cytotoxicity (Fig. 3b and Supplementary Fig. 2a). Since NAC pre-incubation followed by washing still led to lower cellular ROS (data not shown), its failure to prevent AF-induced cytotoxicity suggested that ROS accumulation might not be a key cytotoxic mechanism. 
To further evaluate the role of TrxR inhibition in AF-induced cytotoxicity, we tested if suppression of TrxR expression by siRNA could affect cell viability. A partial silencing of TrxR1 expression by siRNA in Raji cells resulted in approximately $50 \%$ decrease of total TrxR enzyme activity, which was comparable to the degree of TrxR inhibition by AF, did not cause any loss of cell viability (Fig. 3c). Further, siRNA silencing of both TrxR1 and TrxR2 in a more readily transfectable cell line (HCT116) with a 75\% reduction of total TrxR activity still did not induce any loss of cell viability (Fig. 3c). These data together suggest that inhibition of TrxRs was unlike a key event responsible for the cytotoxic effect of AF.

\section{AF induces severe ATP depletion through double impacts on energy metabolism}

To explore the potential mechanisms for AF-induced cytotoxicity in lymphoma cells, we first tested potential impact of AF on cellular energy metabolism, and observed that AF at a relatively low concentration $(1 \mu \mathrm{M})$ could induce a rapid depletion of ATP in JeKo-1 and Raji cells (Fig. 4a and Supplementary Fig. 3). We then examined the effect of NAC on AF-induced ATP depletion. A simultaneous incubation of NAC with AF could prevent ATP depletion, whereas pretreatment of cells with NAC followed by washing before adding AF was unable to prevent ATP deletion (Fig. 4b). These results were consistent with the cytotoxicity data shown in Fig. 3b and Supplementary Fig. 2a and suggested that ROS did not play a key role in AF-induced energy collapse.

To further explore the mechanism for AF-induced ATP depletion, we tested the impact of AF on two main ATP generation pathways, oxidative phosphorylation and glycolysis, using a Seahorse extracellular flux analyzer to measure oxygen consumption rate (OCR) and extracellular acidification rate (ECAR). A substantial decrease of OCR was detected within minutes after addition of AF to JeKo-1 cells (Fig. 4C). This was consistently observed in other lymphoma cells (Supplementary Fig. 4). Concomitantly, a 30\% decrease of ECAR was also observed after AF treatment in lymphoma cell lines (Fig. 4d and Supplementary Fig. 4). Silencing of TrxR expression by siRNA did not affect the effect of AF on oxygen consumption, since the control Raji cells and TrxR-silenced cells exhibited similar degrees of OCR inhibition by AF (Fig. 4e).

We then further examined the impact of AF on mitochondrial respiratory chain activity. Functional analysis revealed that AF caused a significant suppression of respiratory complex II (Fig. 4f) and a moderate decrease in complex III activity (Fig. 4g), whereas no changes in complexes I, IV and V were detected (Supplementary Fig. 5a). Western blot analysis showed no significant changes in expression of any respiratory chain proteins (Supplementary Fig. 5b). We then used specific inhibitors of mitochondrial respiratory complexes in combination with relevant respiratory substrates to analyze the detail changes in each mitochondrial respiratory chain activity. Supplement of the complex II substrate (succinate) resulted in an increase of OCR in the control cells but not in the AF-treated cells (Fig. 4h), indicating that the activity of complex II was inhibited by AF. In contrast, complex I inhibitor (rotenone) and complex III inhibitor (antimycin) caused a decrease of OCR in both control and AF-treated cells (Fig. 4h and Supplementary Fig. 5c), suggesting complexes I and III were not significantly affected by AF. Since the total mitochondrial oxygen consumption represents the electron transport activities from complex 
$\mathrm{I} \rightarrow \mathrm{CoQ} \rightarrow \mathrm{III} \rightarrow \mathrm{IV}$ and complex $\mathrm{II} \rightarrow \mathrm{CoQ} \rightarrow \mathrm{II} \rightarrow \mathrm{IV}(17)$, these data together suggest that the main site inhibited by AF was complex II.

To test if AF inhibition of mitochondrial respiration was responsible for its cytotoxicity, various concentrations of rotenone (a potent mitochondrial respiration inhibitor) were titrated to select a concentration that caused a similar degree of respiratory suppression induced by $1 \mu \mathrm{M} \mathrm{AF}$

(Supplementary Fig. $6 \mathrm{a}$ ). This concentration of rotenone $(0.05 \mu \mathrm{M})$, although potently inhibit respiration, did not cause any significant ATP depletion or cell death (Supplementary Fig. 6b and c). Importantly, the rotenone-treated cells exhibited a significant upregulation of glycolysis whereas the AF-treated cells exhibited a decrease in glycolysis (Fig. 4i), indicating that cells were able to compensate the energy loss from mitochondrial inhibition by rotenone, but not able to do so when they were treated with AF. These data together suggest a possibility that AF might inhibit both mitochondrial respiration and glycolysis leading to severe ATP depletion.

We then tested the impact of AF on several key glycolytic enzymes, including hexokinase (HK), glyceraldehyde phosphate dehydrogenase (GAPDH), pyruvate kinase (PK), and lactate dedydrogenase (LDH). A dose-dependent decrease in GAPDH activity was observed in two lymphoma cell lines treated with AF (Fig. 4j and Supplementary Fig. 7a). In vitro enzyme assay using purified GAPDH showed that AF directly inhibited GAPDH (Fig. 4k), whereas it had no impact on other glycolytic enzymes (Supplementary Fig. 7b-d).

Since pyruvate is the product of glycolysis that functions as a precursor for acetyl CoA production in mitochondria $(18,19)$, it might be possible that the suppression of mitochondrial respiration in AF-treated cells could be a consequence of glycolytic inhibition leading to a depletion of pyruvate. To exam this possibility, we tested whether exogenous pyruvate could rescue the AF-induced respiratory inhibition and cytotoxicity. Addition of pyruvate did not prevent AF-induced mitochondrial dysfunction (Supplementary Fig. 8a), nor did it suppress apoptosis (Supplementary Fig. 8b), indicating that AF-induced mitochondrial dysfunction was not a consequence of GAPDH inhibition.

\section{Normal cells survive AF inhibition of mitochondrial respiration through upregulation of glycolysis}

We then tested the impact of AF on energy metabolism in normal cells. Interestingly, mitochondrial respiration in normal PBMCs and stromal cells was inhibited by AF (Fig. 5a) to similar degrees as observed in cancer cells (Fig. 4c). However, unlike cancer cells whose glycolysis was also inhibited by AF, normal cells exhibited a significant upregulation of glycolysis after AF treatment (Fig. 5b). Such increase in glycolysis seemed able to compensate the decrease of mitochondrial ATP generation, since total cellular ATP did not decrease in the three types of normal cells treated with AF (Fig. 5c). The ability of normal cells to maintain cellular ATP seemed consistent with their ability to survive AF treatment.

\section{Stromal cells promote TrxR1 expression in cancer cells and enhance AF-induced ROS generation}


The finding that AF induced severe ATP depletion could explain its cytotoxic effect against cancer cells, but this could not explain why AF was more effective in killing cancer cells in the presence of stromal cells. In our attempt to explore the underlying mechanisms, we observed that in the presence of stromal cells, AF induced higher ROS generation compared to the cells treated with AF without stromal cells (Supplementary Fig. 9). Such higher ROS was unexpected, since stromal cells normally protect cancer cells by reducing their ROS stress (20). This unexpected observation prompted us to investigate the underlying mechanism. Since AF could inhibit TrxRs and causes ROS increase, we first examined the impact of stromal cells on TrxR expression in cancer cells. Western blot analysis revealed that TrxR1 expression was consistently up-regulated in lymphoma cells when they were co-cultured with stromal cells (Fig. 6a).

The consistent upregulation of TrxR1 by stromal cells led us to speculate its potential role in AF-induced ROS generation. Since the reactive cysteine and selenocysteine residues in the active site of TrxR1 play key roles in transporting electron and mediating the redox cycle during the reduction of thioredoxin (21), we postulate that a reaction of AF with the cysteine or selenocysteine in the active site of TrxR1 would disrupt the electron transport process, leading to a leak of electrons to be captured by molecular oxygen to form superoxide as illustrated in Fig. 6b. As such, AF would turn TrxR1 into a ROS-generating protein, and the higher expression of TrxR1 in cancer cells in stromal environment would generate more ROS in the presence of AF. To test this possibility, we used a luminescence-based in vitro assay to measure superoxide generation in a cell-free system containing purified TrxR1, Trx, NADPH, and AF. Addition of AF

to the complete reaction mixture caused a rapid generation of ROS in a time-dependent manner (red color curve). Removal of AF or TrxR1 from the reaction system led to a substantial decrease in ROS generation rate (Fig. 6b), suggesting that both TrxR1 and AF were required for a sustained production of ROS. Collectively, these data suggest that AF could turn the TrxR1/Trx/NADPH redox system into a ROSgenerating machinery, and the increased expression of TrxR1 in cancer cells in stromal environment renders them more prone to AF-induced ROS generation.

\section{AF exhibits potent therapeutic activity in lymphoma models}

Since AF could effectively kill lymphoma cells in vitro, we further test its therapeutic activity in vivo. In the lymphoma xenograft mouse model bearing lymphoma cells, AF treatment showed a significant therapeutic effect (Fig. 7a). This drug was well tolerated, and the mice exhibited no significant loss of body weight (Fig. 7a). Importantly, there was a significant decrease in both OCR and ECAR in tumor cells isolated from the lymphoma-bearing mice treated with AF (Supplementary Fig. 10), indicating that AF was able to inhibit tumor cell energy metabolism in vivo, similar to that observed in vitro. These data together suggest that AF has promising in vivo therapeutic activity in lymphoma.

\section{Discussion}

As an anti-rheumatoid drug, AF has been used in clinical treatment of patients with certain rheumatoid arthritis for decades. Recent studies show that this compound also has significant anticancer activity, 
and suggest a possibility to repurpose this old drug for new disease indications. However, repurpose of $\mathrm{AF}$ as an anticancer drug has been hindered due in part to unclear mechanisms and undefined advantages over other anticancer drugs. Our study demonstrated that AF was highly effective in killing lymphoma cells, as well as other cancer cells of different tissue origins. One clear advantage of AF is high therapeutic selectivity without significant toxicity to normal cells, and another advantage would be the unique mechanisms of action in impacting energy metabolism leading to severe ATP depletion in cancer cells.

Our study has revealed the unique mechanisms of action responsible for AF's anticancer activity. Inhibition of TrxRs by AF has been known for a long time, and TrxRs are considered the primary targets of AF. It has been suggested that inhibition of TrxR by AF would lead to an increase in ROS, which could cause cellular damage and cancer cell death $(5,22)$. However, our study showed that although AF could indeed inhibit TrxR activity, such inhibition seems unlikely responsible for its cytotoxicity. This conclusion is supported by the following observations: (1) siRNA knockdown of TrxR expression reduced TrxR enzyme activity to the same degree as the inhibition by a toxic concentration of AF, but such TrxR knockdown did not cause any significant loss of cell viability; (2) Knockdown of TrxR expression by siRNA did not affect cellular sensitivity to AF. Lymphoma cells with TrxR knockdown remained sensitive to AF; (3) Antioxidants such as NAC (with wash) did not rescue cell death induced by AF, suggesting the ROS increase due to TrxR inhibition may not be a key event responsible for cytotoxicity.

In our mechanistic study, we discovered that AF induced severe ATP depletion through "double hits" on energy metabolism. Our data showed that AF suppressed mitochondrial respiration via inhibiting complex II of the respiratory chain, and inhibited glycolysis via targeting GAPDH. Since energy metabolism has a high degree of plasticity and inhibition of one metabolic pathway (e.g. mitochondrial oxidative phosphorylation) could be compensated by upregulation of another pathway (e.g. glycolysis), thus the double hits on both pathways are important for AF to cause energy collapse in cancer cells. This notion is supported by the observation that rotenone potently inhibited mitochondrial respiration but did not cause severe ATP depletion nor induced cell death, due to up-regulation of glycolysis. Such compensatory mechanism may also provide a basis to understand the therapeutic selectivity of AF. It is important to note that in all three types of normal cells (PBMC, NKtert, HK), AF could inhibit their mitochondrial respiration but did not cause ATP depletion and cell death, largely due to an upregulation of glycolysis in the normal cells. However, it is still unclear why normal cells were able to up-regulate glycolysis when mitochondrial respiration was inhibited, whereas cancer cells were unable to do so. One potential explanation would be that GAPDH in normal cells is insensitive to AF due the presence of a yet unknown factor that interacts with GAPDH and protects this enzyme from being targeted by AF. Alternatively, normal cells could still up-regulate glycolysis by bypassing the GAPDH-catalyzed steps through channeling proper metabolites into the glycolytic pathway at a step downstream of GAPDH. These possibilities require further investigation in separate studies. Figure $7 \mathrm{~b}$ provides a schematic illustration of the mechanisms of action of auranofin against cancer cells. 
In summary, our study showed that the anti-rheumatic agent AF could effectively kill lymphoma cells through induction of severe ATP depletion by inhibition of mitochondrial respiration and suppression of glycolysis. Our new findings underscore a possibility to repurpose the anti-rheumatic drug AF as a promising anticancer agent for treatment of multiple cancers, especially lymphoma. Based on the new mechanisms of $A F$, we expect that $A F$ could be even more effective in combination with other mitochondria-targeting drugs such as lonidamine and arsenic trioxide (23). These possibilities merit further evaluation in laboratory and clinical setting.

\section{Abbreviations}

AF: auranofin; TrxRs: thioredoxin reductases; ROS: reactive oxygen species; Trx: thioredoxin; MCL: mantle cell lymphoma; DLBCL: diffused large B cell lymphoma; PBMC: peripheral blood mononuclear cells; NAC: $\mathrm{N}$-acetyl-L-cysteine; OCR: oxygen consumption rate; ECAR: extracellular acidification rate; CM: conditional medium; HK: hexokinase; GAPDH: glyceraldehyde phosphate dehydrogenase; PK: pyruvate kinase; LDH: lactate dedydrogenase.

\section{Declarations}

\section{Ethics approval and consent to participate}

The study using human lymphoma samples was reviewed and approved by Committee for Ethical Review of Research involving Human Subjects of Sun Yat-Sen University.

\section{Consent for publication}

Not applicable

\section{Availability of data and material}

The datasets used and/or analyzed during this study are included in this published article, and its supplementary information files.

\section{Competing interests}

The authors declare that they have no competing interests.

\section{Funding}

This work was supported in part by grants from National Key R\&D Program of China (2018YFC0910203), the Key R\&D Program of Guangdong Province (2019B020226001), National Natural Science Foundation of China (No. 81430060区No.81502573, No 81972575), Guangzhou Technology Program (No. 201508020250, No. 201504010038, No. LCY201317, No 201804010483), Natural Science Foundation of 
Guangdong Province (No. 2014A030310421) and Young Teacher Fund of Sun Yat-Sen University (No. 16ykpy20).

\section{Authors' contributions}

M.Y., P.L., J.L., and P.H. designed the project; M.Y., P.L., H.C., J.L., J.Y., Y.H., W.L., P.C., G.H., and W.J. performed research; M.Y., P.L., S.W., J.L., W.J. and P.H. analyzed and interpreted the data; M.Y, P.L., J.L. and P.H. wrote the paper.

Mengqi Yang and Panpan Liu contributed equally to this article.

\section{Acknowledgements}

The authors thank the grant support from the funding agencies listed in the Funding section.

\section{References}

1. Waghray $M$, Yalamanchili M, Dziubinski M, Zeinali M, Erkkinen $M$, Yang H, et al. GM-CSF Mediates Mesenchymal-Epithelial Cross-talk in Pancreatic Cancer. Cancer Discov. Aug;6(8):886-99.

2. McGranahan N, Swanton C. Clonal Heterogeneity and Tumor Evolution: Past, Present, and the Future. Cell. 2017 Feb 9;168(4):613-28.

3. Debnath A, Parsonage D, Andrade RM, He C, Cobo ER, Hirata K, et al. A high-throughput drug screen for Entamoeba histolytica identifies a new lead and target. Nature medicine. 2012 Jun;18(6):956-60.

4. Fath MA, Ahmad IM, Smith CJ, Spence J, Spitz DR. Enhancement of carboplatin-mediated lung cancer cell killing by simultaneous disruption of glutathione and thioredoxin metabolism. Clinical cancer research : an official journal of the American Association for Cancer Research. 2011 Oct 01;17(19):6206-17.

5. Fiskus W, Saba N, Shen M, Ghias M, Liu J, Gupta SD, et al. Auranofin induces lethal oxidative and endoplasmic reticulum stress and exerts potent preclinical activity against chronic lymphocytic leukemia. Cancer research. 2014 May 01;74(9):2520-32.

6. Gandin V, Fernandes AP, Rigobello MP, Dani B, Sorrentino F, Tisato F, et al. Cancer cell death induced by phosphine gold(I) compounds targeting thioredoxin reductase. Biochemical pharmacology. 2010 Jan 15;79(2):90-101.

7. Omata Y, Lewis JB, Lockwood PE, Tseng WY, Messer RL, Bouillaguet S, et al. Gold-induced reactive oxygen species (ROS) do not mediate suppression of monocytic mitochondrial or secretory function. Toxicology in vitro : an international journal published in association with BIBRA. 2006 Aug;20(5):625-33.

8. Rigobello MP, Folda A, Dani B, Menabo R, Scutari G, Bindoli A. Gold(I) complexes determine apoptosis with limited oxidative stress in Jurkat T cells. European journal of pharmacology. 2008 Mar 17;582(1-3):26-34. 
9. Liu J, Chen G, Feng L, Zhang W, Pelicano H, Wang F, et al. Loss of p53 and altered miR15-a/16-1short right arrowMCL-1 pathway in CLL: insights from TCL1-Tg:p53(-/-) mouse model and primary human leukemia cells. Leukemia. 2014 Jan;28(1):118-28.

10. Cramer SL, Saha A, Liu J, Tadi S, Tiziani S, Yan W, et al. Systemic depletion of L-cyst(e)ine with cyst(e)inase increases reactive oxygen species and suppresses tumor growth. Nature medicine. 2017 Jan;23(1):120-7.

11. Oppermann S, Ylanko J, Shi Y, Hariharan S, Oakes CC, Brauer PM, et al. High-content screening identifies kinase inhibitors that overcome venetoclax resistance in activated CLL cells. Blood. 2016 Aug 18;128(7):934-47.

12. Jitschin R, Braun M, Qorraj M, Saul D, Le Blanc K, Zenz T, et al. Stromal cell-mediated glycolytic switch in CLL cells involves Notch-c-Myc signaling. Blood. 2015 May 28;125(22):3432-6.

13. Troeger A, Johnson AJ, Wood J, Blum WG, Andritsos LA, Byrd JC, et al. RhoH is critical for cellmicroenvironment interactions in chronic lymphocytic leukemia in mice and humans. Blood. 2012 May 17;119(20):4708-18.

14. Hwang-Bo H, Jeong JW, Han MH, Park C, Hong SH, Kim GY, et al. Auranofin, an inhibitor of thioredoxin reductase, induces apoptosis in hepatocellular carcinoma Hep3B cells by generation of reactive oxygen species. General physiology and biophysics. 2017 Apr;36(2):117-28.

15. Liu N, Li X, Huang H, Zhao C, Liao S, Yang C, et al. Clinically used antirheumatic agent auranofin is a proteasomal deubiquitinase inhibitor and inhibits tumor growth. Oncotarget. 2014 Jul 30;5(14):545371.

16. Kim NH, Park HJ, Oh MK, Kim IS. Antiproliferative effect of gold(I) compound auranofin through inhibition of STAT3 and telomerase activity in MDA-MB 231 human breast cancer cells. BMB reports. 2013 Jan;46(1):59-64.

17. Hu Y, Lu W, Chen G, Wang P, Chen Z, Zhou Y, et al. K-ras(G12V) transformation leads to mitochondrial dysfunction and a metabolic switch from oxidative phosphorylation to glycolysis. Cell research. 2012 Feb;22(2):399-412.

18. Gray LR, Sultana MR, Rauckhorst AJ, Oonthonpan L, Tompkins SC, Sharma A, et al. Hepatic Mitochondrial Pyruvate Carrier 1 Is Required for Efficient Regulation of Gluconeogenesis and WholeBody Glucose Homeostasis. Cell metabolism. 2015 Oct 06;22(4):669-81.

19. Petersen KF, Befroy DE, Dufour S, Rothman DL, Shulman GI. Assessment of Hepatic Mitochondrial Oxidation and Pyruvate Cycling in NAFLD by (13)C Magnetic Resonance Spectroscopy. Cell metabolism. 2016 Jul 12;24(1):167-71.

20. Zhang W, Trachootham D, Liu J, Chen G, Pelicano H, Garcia-Prieto C, et al. Stromal control of cystine metabolism promotes cancer cell survival in chronic lymphocytic leukaemia. Nature cell biology. 2012 Mar;14(3):276-86.

21. Zhong L, Arner ES, Holmgren A. Structure and mechanism of mammalian thioredoxin reductase: the active site is a redox-active selenolthiol/selenenylsulfide formed from the conserved cysteine- 
selenocysteine sequence. Proceedings of the National Academy of Sciences of the United States of America. 2000 May 23;97(11):5854-9.

22. Wang H, Bouzakoura S, de Mey S, Jiang H, Law K, Dufait I, et al. Auranofin radiosensitizes tumor cells through targeting thioredoxin reductase and resulting overproduction of reactive oxygen species. Oncotarget. 2017 May 30;8(22):35728-42.

23. Sordet O, Rebe C, Leroy I, Bruey JM, Garrido C, Miguet C, et al. Mitochondria-targeting drugs arsenic trioxide and lonidamine bypass the resistance of TPA-differentiated leukemic cells to apoptosis. Blood. 2001 Jun 15;97(12):3931-40.

\section{Figures}


Figure 1

a

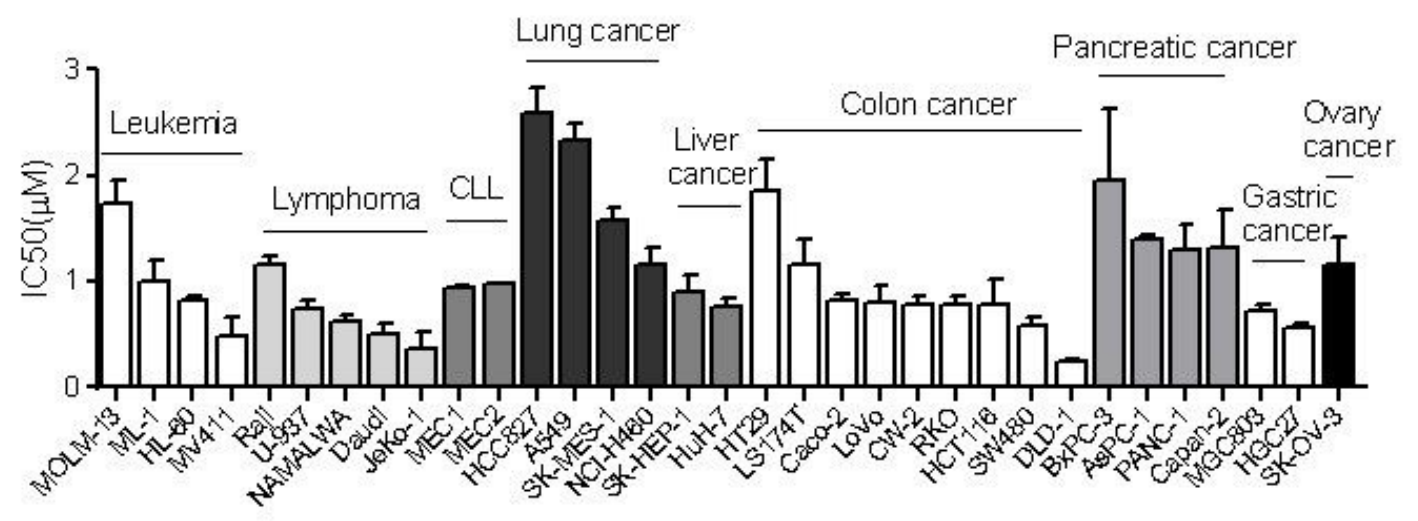

b

C

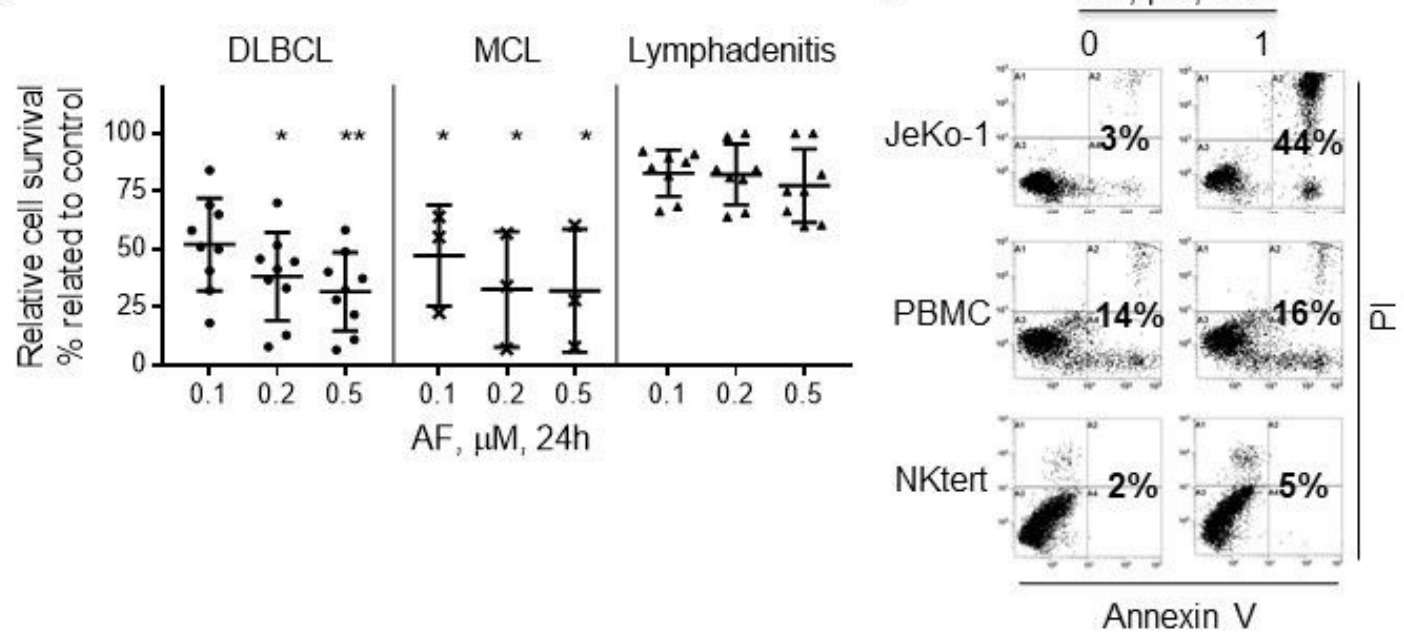

Figure 1

Cytotoxic effect of AF on various types of cancer cells in vitro. (a) IC50 values of AF in various cancer cell lines. Cells were exposed to AF for $72 \mathrm{~h}$ and cell viability was measured by MTS assay $(n=3)$. (b) Effect of AF on primary cells from lymph node of patients with mantle cell lymphoma $(M C L, n=3)$, diffused large $B$ cell lymphoma (DLBCL, $n=9)$, and lymphadenitis $(n=8)$ measured by annexin V-PI assay. (c)Represented cell viability analysis in AF-treated JeKo-1, PBMC and NKtert cells measured by annexin V- 
PI assay $(n=3)$, representative data are shown. The number in each panel shows \% of dead cells (left panel). Effects of AF on the viability of peripheral blood mononuclear cells (PBMC) and stromal cells (NKtert and HK cells), analyzed by annexin V-PI assay (right panel; $n=3$ ). For a-c, data are presented as mean $\pm S D ;{ }^{*} p<0.05,{ }^{* *} p<0.01$.

Figure 2

a
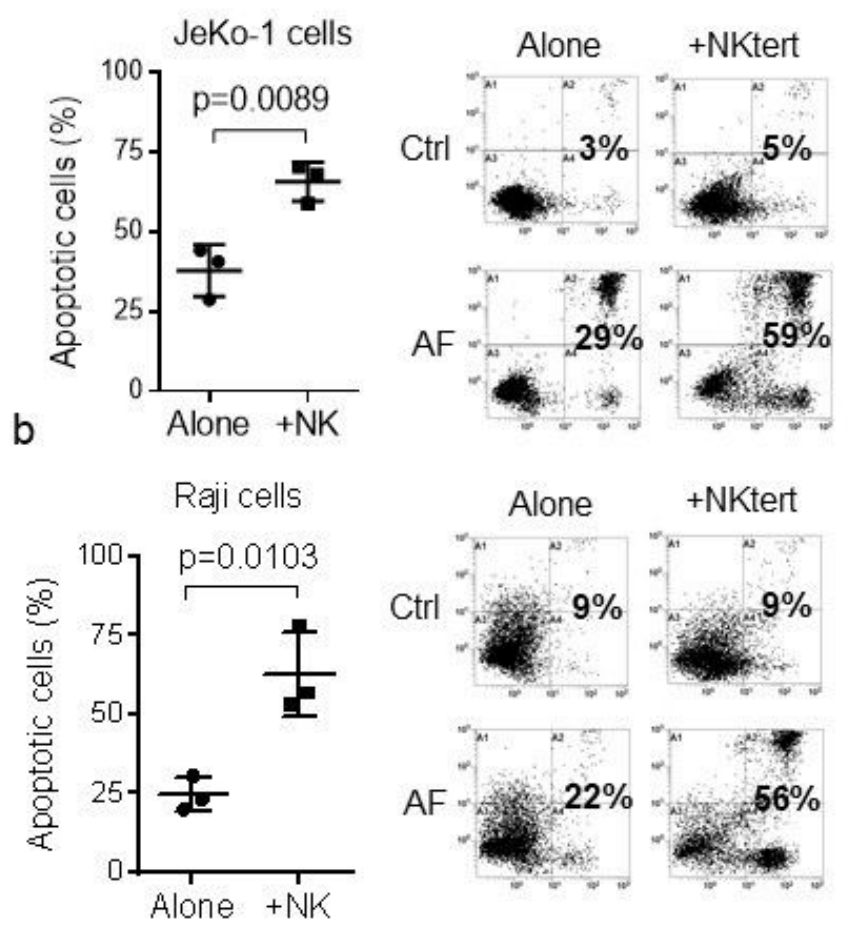

Figure 2 
Enhanced anticancer activity of AF in the presence of stromal cells. ( $a$ and b) Viability of JeKo-1 or Raji cells treated with 0.5 or $1 \mu \mathrm{M} \mathrm{AF}$ for $24 \mathrm{~h}$ in the presence or absence of stromal NKtert cells, measured by annexin V-PI assay $(n=3)$. Representative data were shown. For $a-b$, data are presented as mean $\pm S D$.

Figure 3

a

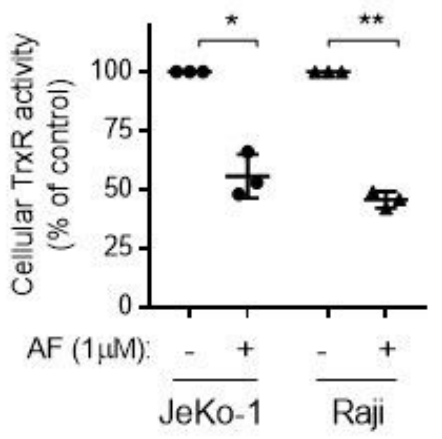

b

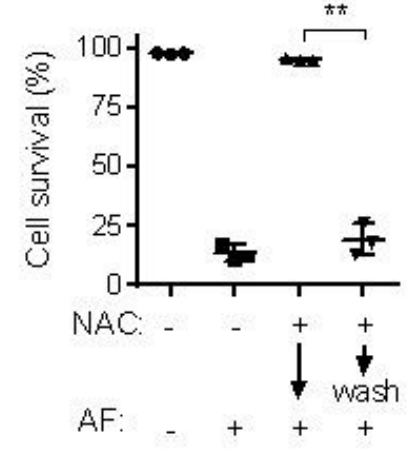

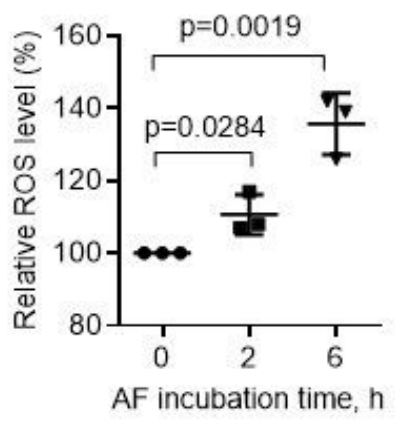

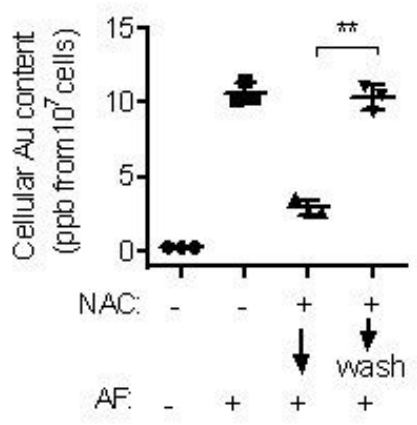

C
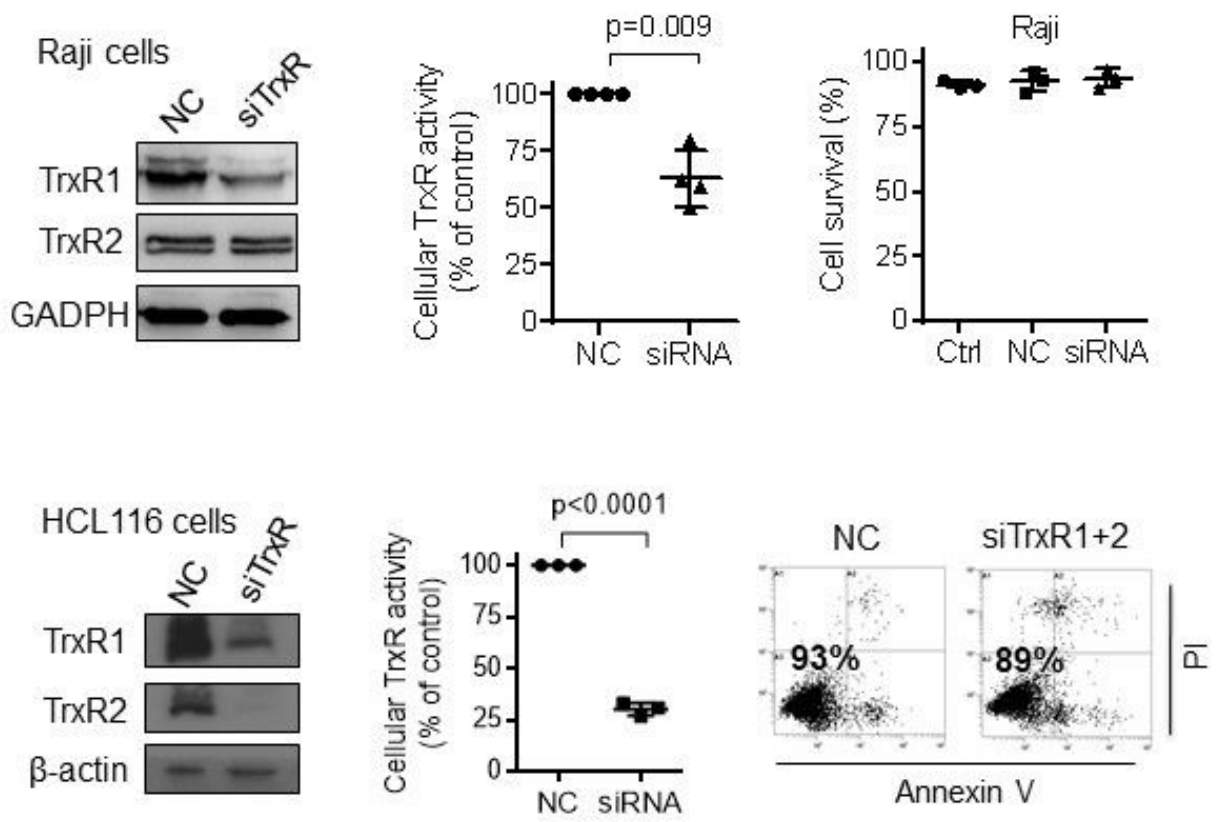

Figure 3

Inhibition of TrxRs by AF and its impact on cell viability. (a) Cellular TrxR activity in JeKo-1 and Raji cells after treatment with $\mathrm{AF}(\mathrm{n}=3)$ (left panel). ROS levels in JeKo-1 cells detected by staining with 
hydroethidine (Het) before and after AF treatment ( $n=3$ ) (right panel). (b) Impact of NAC on cell survival and cellular Au content in AF-treated cells. JeKo-1 cells were pretreated with $2 \mathrm{mM} \mathrm{NAC}$ for $4 \mathrm{~h}$, then washed twice with fresh medium or not as indicated, followed by $1 \mu \mathrm{M} \mathrm{AF}$ treatment for $20 \mathrm{~h}$ or $2 \mathrm{~h}$. Cell viability was measured by annexin V-PI assay; intracellular Au content was analyzed by ICP-MS( $n=3)$. (c) Western blot analysis of TrxR1 and TrxR2 in Raji cells and HCT116 cells after electroporation with siRNA for48 $h(n=3)$. Representative data were shown. TrxR1 and TrxR2 knockdown by siRNA led to a decrease in cellular TrxR activity and cell survival, $n=3$ independent experiments. Representative data were shown. The number in each panel shows $\%$ of viable cells. For a-c, data are presented as mean $\pm S D ; * p<0.05$, ** $p<0.01$. 
Figure 4

a

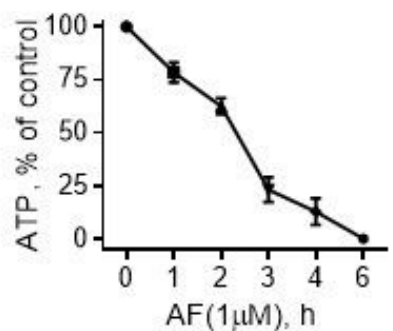

d

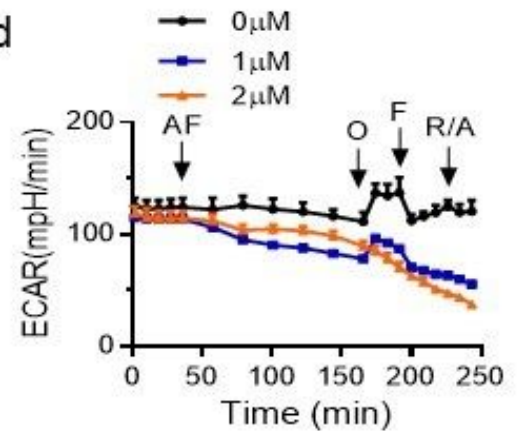

b

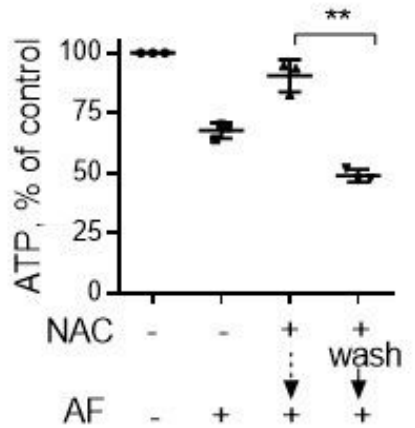

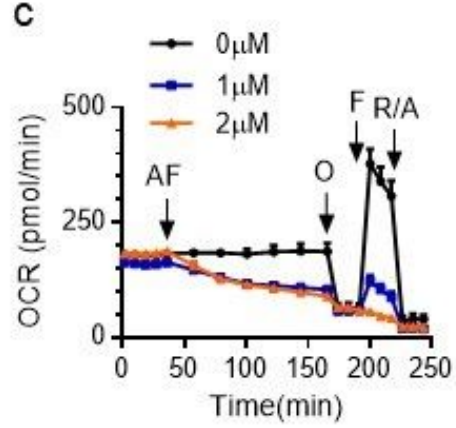

e

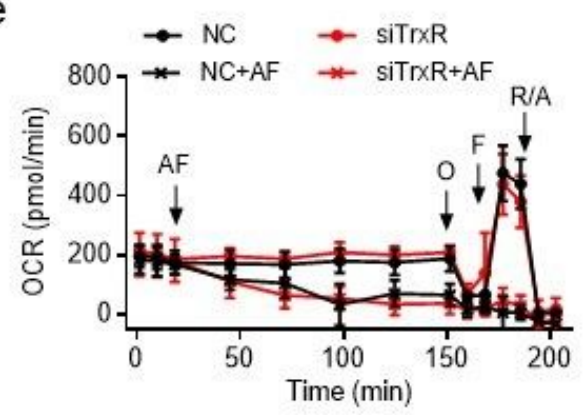

f

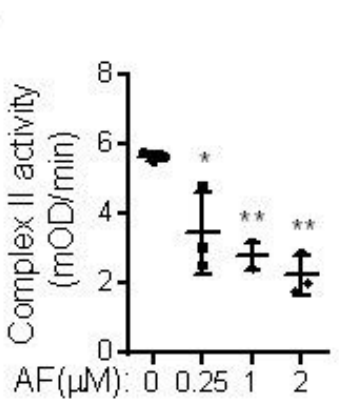

g

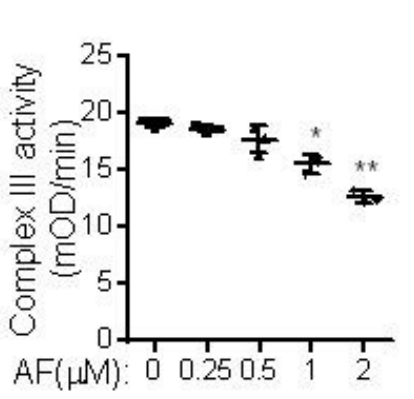

$\mathrm{h} \rightarrow \mathrm{Ctrl}$

$\rightarrow \mathrm{AF} 1 \mu \mathrm{M} 30 \mathrm{~min}$

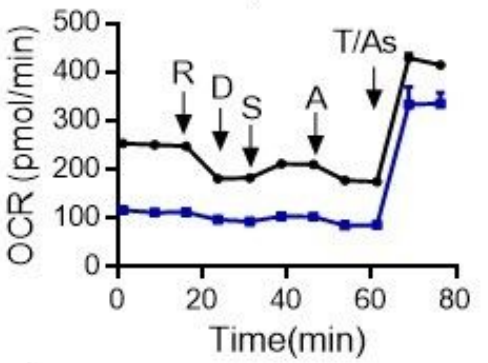

i
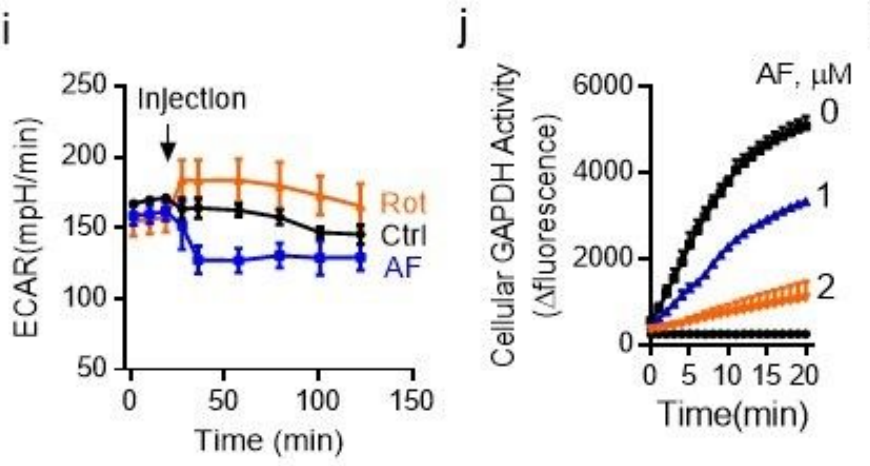

k

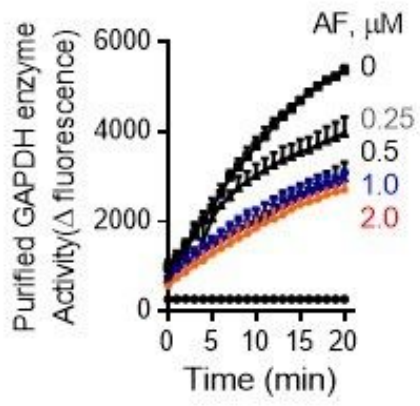

\section{Figure 4}

Induction of severe ATP depletion by AF through inhibition of mitochondrial respiration and inhibition of GAPDH. (a) ATP levels in JeKo-1 cells treated with AF for the indicated time periods $(n=3)$. (b) Effect of NAC on intracellular ATP levels in AF-treated cells $(n=3)$. (c and d) Real-time measurement of oxygen consumption rate (OCR) and extracellular acidification rate (ECAR) in JeKo-1 cells, using the Seahorse

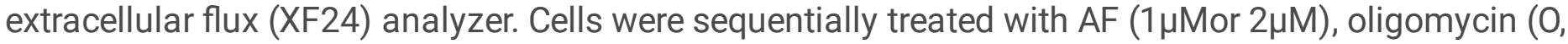


$1 \mu \mathrm{M}), \operatorname{FCCP}(F, 1 \mu \mathrm{M})$ and rotenone/antimycin $A(R / A, 0.5 \mu \mathrm{M})(n=4)$. (e) Effect of siRNA silencing of TrxR expression on OCR in AF-treated Raji cells. ( $f$ and $g$ ) Inhibition of mitochondrial complex II ( $f$ ) and complex III $(\mathrm{g})$ by AF $(\mathrm{n}=3)$. (h)Real-time measurement of OCR in JeKo-1 cells pre-treated with or without $1 \mu \mathrm{M} \mathrm{AF}$ for $30 \mathrm{~min}(n=5)$. Cells were then sequentially treated with rotenone $(R, 2 \mu M)$, digitonin $(D, 15$ $\mu \mathrm{g} / \mathrm{ml})$, succinate $(\mathrm{S}, 10 \mathrm{mM})$, antimycin $\mathrm{A}(\mathrm{A}, 2 \mu \mathrm{M})$, TMPD/ascorbate (T/As, $100 \mu \mathrm{M} / 5 \mathrm{mM})$ as indicated. (i) Comparison of ECAR in JeKo-1 cells treated with rotenone or AF ( $n=4$ cell culture replicates at each dose). ( $\mathrm{j}$ and $\mathrm{k}$ ) Dose-dependent inhibition of GAPDH enzyme activity by AF, detected in JeKo-1 cell lysates $(j)$ or in assay with purified GAPDH $(k), n=3$ independent experiments. For a-k, data are presented as mean $\pm S D ;{ }^{*} p<0.05,{ }^{*} \mathrm{p}<0.01$. 
Figure 5
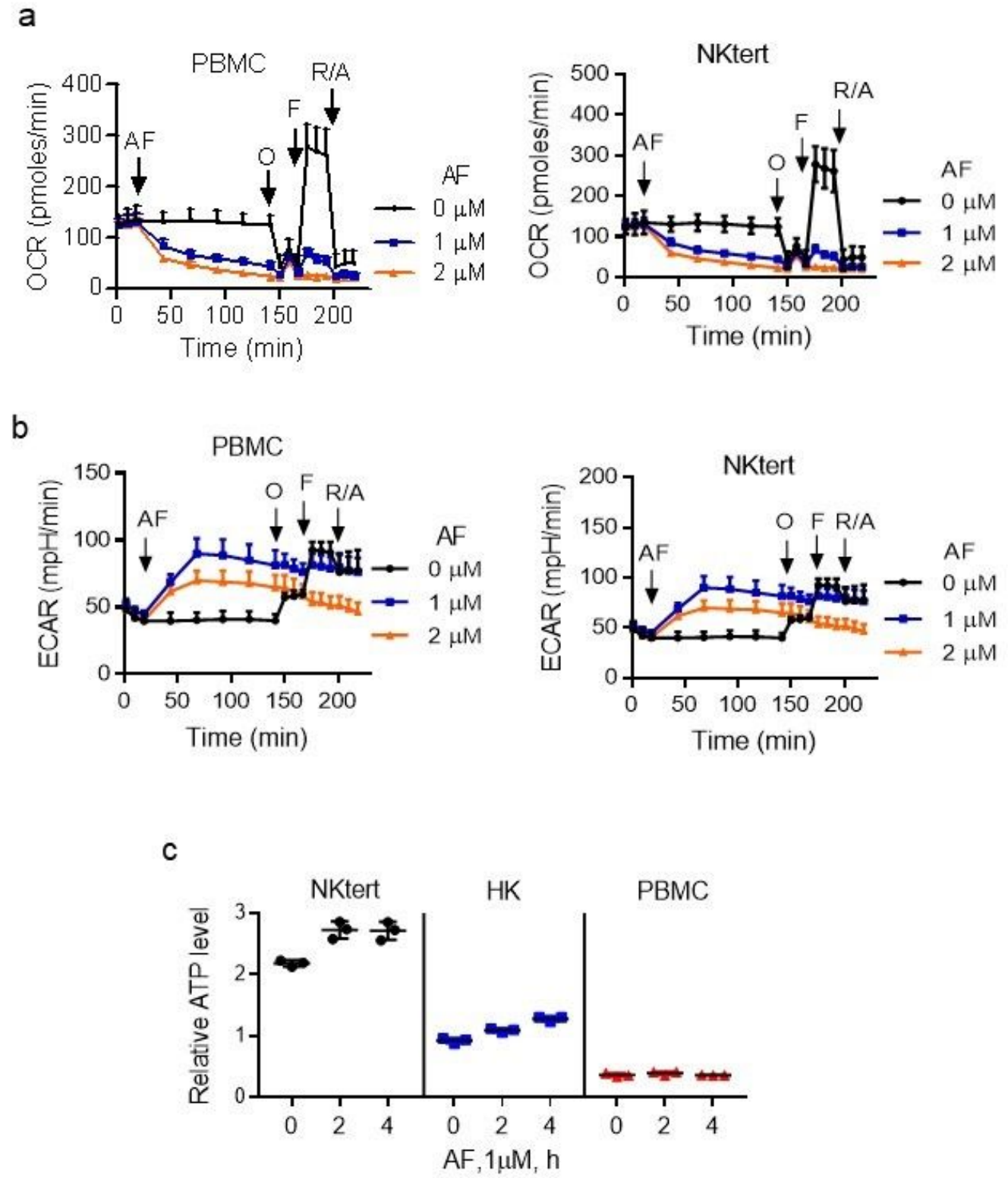

\section{Figure 5}

Impact of AF on energy metabolism in normal cells. (a-b) Real-time measurement of OCR and ECAR using the Seahorse extracellular flux XF24 analyzer $(n=4)$. The PBMC cells were sequentially treated with AF ( 1 or $2 \mu \mathrm{M})$, oligomycin $(0,1 \mu \mathrm{M}), \mathrm{FCCP}(\mathrm{F}, 1 \mu \mathrm{M})$ and rotenone/antimycin $A(R / A, 0.5 \mu \mathrm{M})$. (c) Effect of $A F$ on intracellular ATP levels in stromal cells HK, NKtert, and PBMC $(n=3)$. For a-c, data are shown as mean \pm SD. 
Figure 6

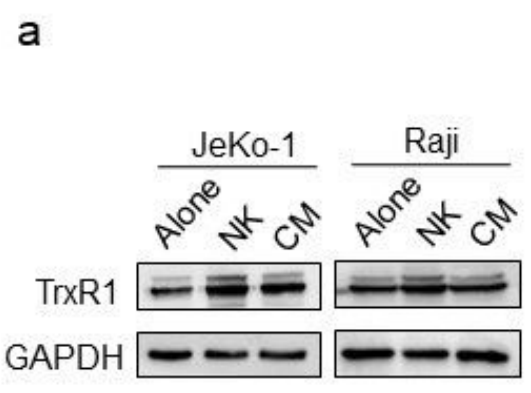

b
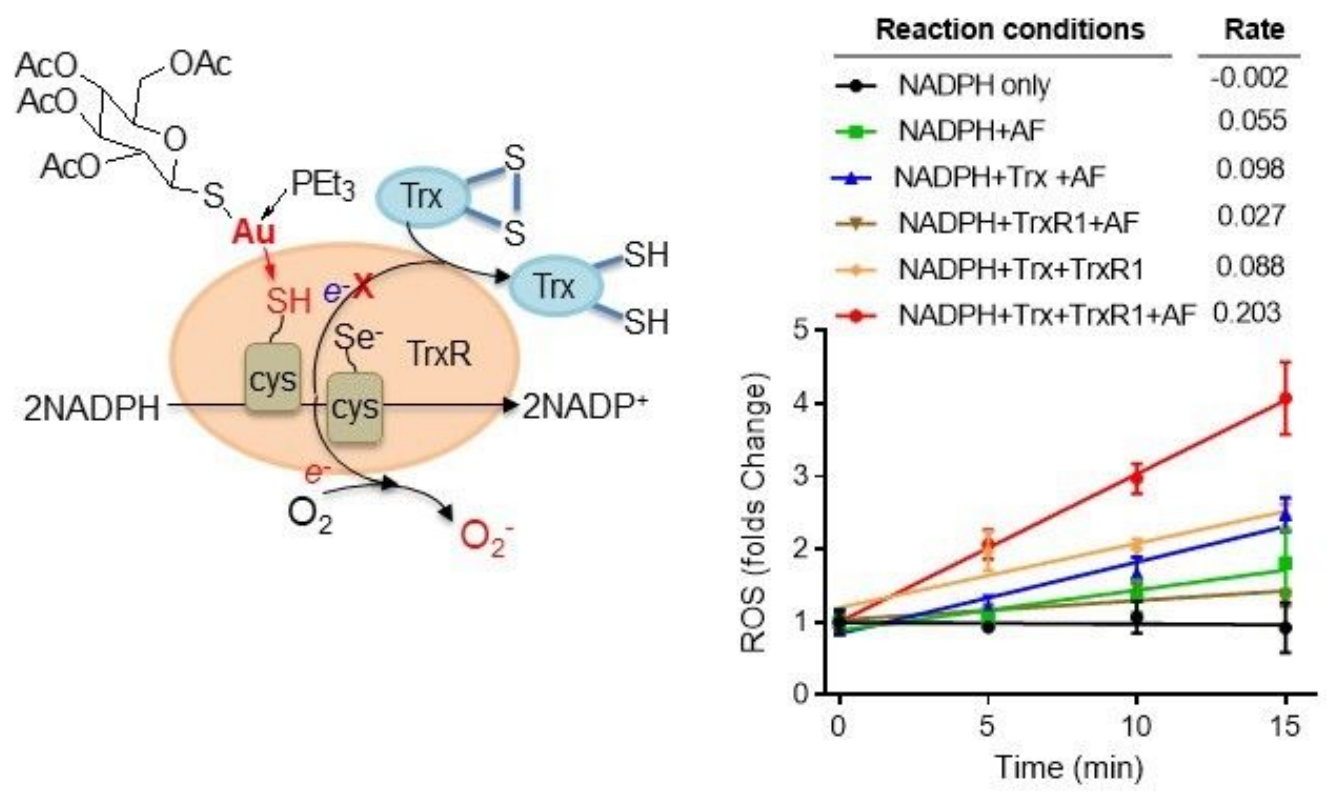

\section{Figure 6}

Stromal cells promote TrxR1 expression in lymphoma cells leading to elevated ROS generation in the presence of AF. (a) Effect of stromal cells on TrxR1 expression in lymphoma cells, detected by western blotting. Representative data of at least three experiments were shown. (b) A schematic diagram showing AF-induced ROS generation by interaction with TrxR1. The interaction of AF with the cysteine or selenocysteine residuals in the active site of TrxR1disrupts the electron transport process, leading to a 
leak of electrons, which could be captured by oxygen to produce superoxide. Measurement of ROS in a cell-free system, detected by luminescence in the presence of NADPH, NADPH + AF, NADPH + AF+ Trx, $\mathrm{NADPH}+\mathrm{AF}+\operatorname{TrxR} 1, \mathrm{NADPH}+\operatorname{Trx}+\operatorname{TrxnR1}$, or NADPH + AF + Trx +TrxnR1 as indicated $(n=3)$. For a and $b$, data are presented as mean \pm SD.

Figure 7

a
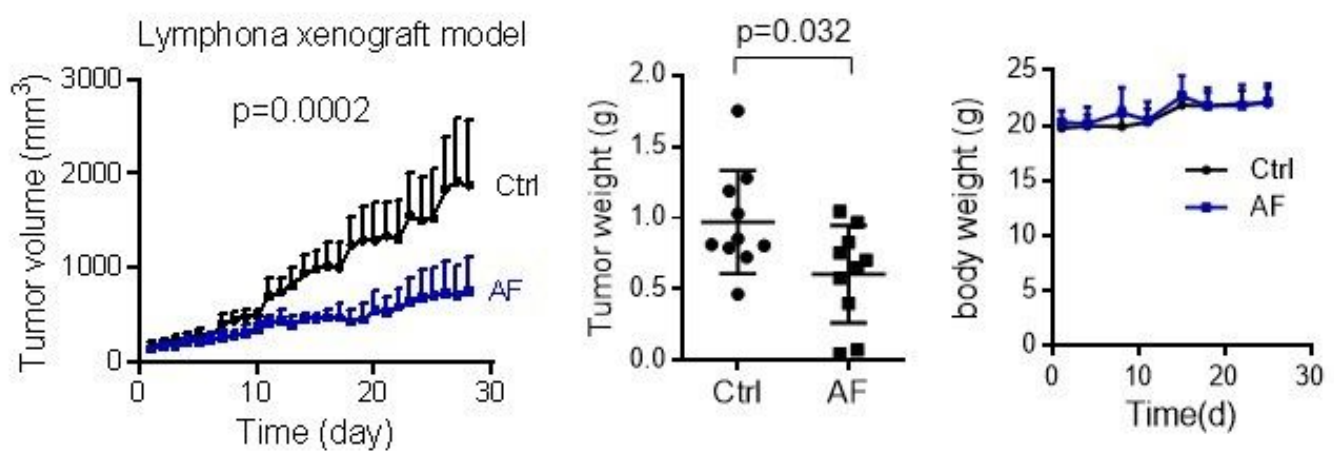

b

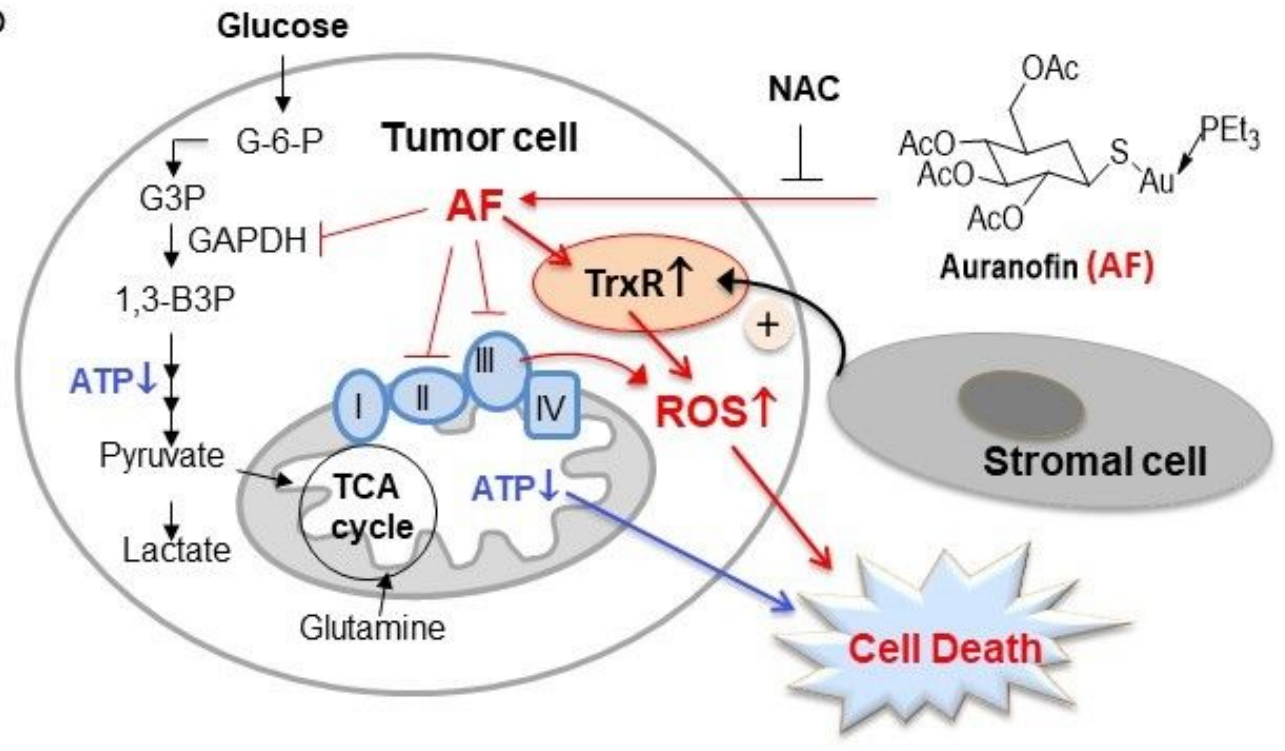

Figure 7 
In vivo therapeutic activity of AF in lymphoma xenograft model. (a) Nude mice bearing JeKo-1 tumors were treated with normal saline or AF (10 mg/kg/day i.p.) $5 \mathrm{~d}$ per week for four weeks ( $\mathrm{n}=10$ per group). Tumor volume and body weight were shown. Results are presented as mean \pm SD, $p$ values were determined by Student's t test. (b) Schematic illustration of the proposed mechanisms of action of AF against cancer cells.AF induces severe ATP depletion by double impacts on glycolysis and mitochondrial respiration through inhibition of GAPDH and suppression of complexes II and III. The thiol-antioxidant NAC rescued AF-induced cell death by conjugating with AF and preventing the drug uptake by cancer cells.

\section{Supplementary Files}

This is a list of supplementary files associated with this preprint. Click to download.

- SupplementaryFigures.docx 